\title{
COMPOSIÇÃO CENTESIMAL E NUTRICIONAL DA MACADÂMIA (Macadamia integrifolia Maiden \& Betch) E ANÁLISE DO SEU PERFIL LIPÍDICO RELACIONADO À TERAPÊUTICA
}

\author{
MACADAMIA'S (Macadamia integrifolia Maiden \& Betch) CENTESIMAL AND \\ NUTRITIONAL COMPOSITION AND PROFILE ANALYSIS RELATED TO \\ TERAPHEUTICS
}

\author{
FEDALTO, M. B. ${ }^{1}$; STOFELLA, N. C. F. ${ }^{1}$; GOMES, D.L. ${ }^{1}$; MAAS, N. C. ${ }^{1}$; MUNOZ, M. \\ M. ${ }^{1}$; SILVA, M. S. ${ }^{2}$; FLORENTINO, I. ${ }^{3}$; BALBI, M. E. ${ }^{4}$
}

1 - Docentes do Curso de Farmácia, UFPR.

2 - Docente do Curso de Nutrição, UniBrasil.

3 - Farmacêutica, Departamento de Farmácia, UFPR.

4 - Professora da disciplina de Bromatologia, Departamento de Farmácia, UFPR.

Autor para correspondência: bromatologia.ufpr@gmail.com

\section{RESUMO:}

A macadâmia (Macadamia integrifolia Maiden \& Betch) é uma noz considerada nobre pelo seu custo relativamente alto e suas possíveis propriedades funcionais relacionadas à redução dos riscos de doenças cardiovasculares. Além disso, este grão apresenta importância na indústria farmacêutica, pois, o óleo extraído da noz, é de grande valia no desenvolvimento de cremes hidratantes e shampoos, já que tem afinidade com as proteínas da pele e dos cabelos. Com o objetivo de se conhecer e comparar com dados da literatura foram realizadas análises baseadas em métodos de referência. Os resultados foram obtidos com base em uma porção de $15 \mathrm{~g}$, que equivale à medida caseira de uma colher de sopa. A investigação da umidade teve como resultado $1,10 \mathrm{~g}$, valor não condizente com a literatura. Já os resultados de proteínas $(1,40 \mathrm{~g})$, lipídeos $(10,56 \mathrm{~g})$, carboidratos $(1,10 \mathrm{~g})$, fibras $(0,68 \mathrm{~g})$, minerais $(0,16 \mathrm{~g})$ e em $\mathrm{kcal}(105,01)$ em um total de $15 \mathrm{~g}$ de amostra foram muito próximos aos valores encontrados na literatura.

PALAVRAS-CHAVE : Macadâmia; doenças cardiovasculares; ácidos graxos;

\section{ABSTRACT:}

Macadamia (Macadamia integrifolia Maiden \& Betch) is a considerably noble nut for its relatively high cost and its functional properties possible related to the reduction of the cardiovascular diseases risks. Moreover, this grain presents importance in the pharmaceutical industry, therefore, the extracted oil of the nut, is of great value in the development of moisturizers and shampoos creams, since it has affinity with proteins of the skin and the hair. Aiming to meet and compare with literature data analysis were made based on reference methods. The results had been gotten based on a portion of $15 \mathrm{~g}$, that it is equivalent to the measure of a soup spoon. The inquiry of the humidity had as resulted $1,10 \mathrm{~g}$, different from literature. On the other side, the protein results $(1,40 \mathrm{~g})$, lipid $(10,56 \mathrm{~g})$, carbohydrate $(1,10 \mathrm{~g})$, staple fibres $(0,68 \mathrm{~g})$, minerals $(0,16 \mathrm{~g})$ and $\mathrm{kcal}(105,01)$ in a total of $15 \mathrm{~g}$ of sample had been very next to the values found in literature.

KEY-WORDS Macadamia nuts; cardiovascular diseases; fat acids; 


\section{INTRODUÇÃO}

A macadâmia (Macadamia integrifolia Maiden \& Betch) é uma espécie de noz, originária da Austrália, com alto teor de ácidos graxos insaturados que se comportam como antioxidantes. O conhecimento desta propriedade é importante, pois possibilita a aplicação destes antioxidantes nos mecanismos que retardam o envelhecimento e na proteção do sistema cardiovascular. Outra investigação relevante é a diminuição dos níveis de colesterol no sangue, através do equilibrio entre os níveis de colesterol HDL e LDL (SANTOS; BORTO-LOZO, 2008).

Além do possível uso como alimento funcional, o óleo proveniente da macadâmia possui muitos usos na indústria cosmetológica. Sendo ainda altamente custosa a retirada deste óleo, um melhor conhecimento de técnicas para isto faz-se proveitosa para uma maior acessibilidade deste produto na indústria de cosméticos, possibilitando avanços na produção e desenvolvimento de novos produtos cosmetológicos (BEUCHAT; WORTHINGTON, 2007).

\section{REVISÃO DE LITERATURA}

\subsection{Macadamia - Aspectos gerais}

A nogueira macadâmia (Macadamia integrifolia Maiden \& Betch) é uma planta arbórea com folhas lisas de bordos ondulados, de coloração acinzentada quando jovens. Os frutos são arredondados, casca avermelhada com polpa creme-esverdeada, carnosa, que encerra uma noz comestível. A noz tem um leve sabor amanteigado, podendo ser consumida crua ou cozida depois de seca. Torrada, é muito apreciada como aperitivo. A macadâmia frutifica de fevereiro a maio (SACRAMENTO, 1999).

Ela pode demorar entre 12 e 15 anos para chegar à sua máxima produtividade. A partir daí, não pára mais, podendo ter um século de idade e serem ainda muito produtivas (SACRAMENTO, 1999).

\subsection{História}

A macadâmia é originária das províncias de New South Wales e de Queensland. Hoje em dia, é produzida, especialmente na Austrália e no Havaí, e em menor escala na África, na América Central e na Califórnia. Na América do Sul, a planta encontrou boas condições de aclimatação na vasta área que vai desde o sul da Bahia, no Brasil, até o 
Uruguai (PAULINO; MARCHINI, 1998).

Grandes plantações de macadâmia estão instaladas nos Estados de São Paulo, Minas Gerais, Espírito Santo e Bahia. O fato de a Austrália possuir a mesma latitude que as terras brasileiras explica o porquê de a macadâmia ter se adaptado tão bem ao país (DIERBERGER; NETTO, 1985).

\subsection{Cultivo}

Desenvolve-se em climas tropicais e subtropicais com latitudes entre $25^{\circ}$ a $35^{\circ}$, e altitudes entre 100 a 1.200 m. A condição ideal ocorre em solos com profundidade de $1 \mathrm{~m}$, bem drenados e ricos em matéria orgânica. Quanto à temperatura, a melhor situa-se em torno de $25^{\circ} \mathrm{C}$. No inverno requer temperaturas noturnas de $15-18^{\circ} \mathrm{C}$ como estímulo ao florescimento. Altas temperaturas reduzem o crescimento vegetativo, aumentam a queda prematura de frutos, diminuem o tamanho e o acúmulo de óleo (QUEEN NUT MACADAMIA, 2006).

A precipitação necessária é de no mínimo $1.250 \mathrm{~mm}$ anuais bem distribuídos. Locais com alta umidade relativa no período da florada (julho a setembro) devem ser evitadas. As baixas temperaturas somadas a um inverno chuvoso favorecem a ocorrência de doenças como antracnose. Por causa das chuvas, o verão é a melhor época para 0 plantio (QUEEN NUT MACADAMIA, 2006).

A nogueira macadâmia é sensível a ventos, por possuir um sistema radicular superficial. Os ventos intensos podem diminuir significativamente o crescimento das árvores, principalmente as mais jovens (QUEEN NUT MACADAMIA, 2006).

\subsection{Perfil lipídico e saúde}

Segundo Freitas; Naves (2010), sobre a macadâmia é importante ressaltar é a relação ácidos graxos $\omega-6: \omega-3$ visto que, as maiores causas de mortalidade atualmente estão relacionadas com as doenças cardiovasculares, e estudos indicam que essa relação é favorável a redução do risco destas patologias pois, credita-se ao consumo de gordura $\omega-3$, a melhora no perfil lipídico plasmático, a redução dos processos inflamatórios, propriedades antitrombóticas e a redução das síndromes plurimetabólicas que incluem diabetes, obesidade, hipertensão, entre outros. Isso é baseado em evidências que sugerem que os ácidos graxos $\omega-3$, metabolicamente, diminuem a produção hepática de triacilglicerol e apolipoproteína $B$, constituintes das lipoproteínas de densidade muito baixa (VLDL) 
(SANTOS; BORTO-LOZO, 2008).

\subsection{Doenças cardiovasculares e hipertensão no paraná}

As doenças cardiovasculares (DCV) constituem-se nas doenças isquêmicas do coração (DIC) e as cerebrovasculares (AVC). As complicações estão relacionadas com a formação de placas de gorduras nas paredes das artérias que, associadas a processo inflamatório de endotélio e ao aumento da pressão arterial, evoluem para obstrução ou rompimento da artéria. A obstrução ao fluxo sanguíneo e conseqüente insuficiência de oxigenação dos tecidos, leva a degeneração e morte do tecido muscular cardíaco, ou cerebral, ou em qualquer outro tecido do organismo (SESA, 2008).

Os principais fatores de risco para as doenças cardiovasculares são: os erros alimentares, as dislipidemias (particularmente a hipercolesterolemia com alteração na relação entre HDL e LDL colesterol), a hipertensão arterial sistêmica, o diabetes mellitus, o estresse, o tabagismo, o sedentarismo, a obesidade e sua distribuição corporal, a hipertorfia ventricular esquerda, os distúrbios relacionados com o fibrinogênio, entre outros mais discutidos, podendo ser classificados como evitáveis ou inevitáveis (SESA, 2008).

\subsubsection{Mortalidade por doenças cardiovasculares no Paraná}

Em 2004 as doenças cardiovasculares levaram a óbito mais de 20 mil pessoas em todo o Estado. As principais causas de morte foram pelas doenças isquêmicas do coração e doenças cerebrovasculares (SESA, 2008).

Quanto a morbidade, dentre as 96.658 internações hospitalares devidas a doenças cardiovasculares, as insuficiências cardíacas predominam com 31,5\% dessas internações, com 26,9\% estão as cerebrovasculares e as doenças isquêmicas do coração, correspondendo a $20 \%$ das internações por DCV (SESA, 2008).

\subsubsection{Situação epidemiológica da hipertensão arterial}

A hipertensão arterial é doença de relativa gravidade, em decorrência de sua cronicidade e de sua evolução para lesões de órgãos alvos, especialmente as cardiopatias, arteriopatias e doença renal. Segundo a Organização Mundial de Saúde, a nível global a hipertensão arterial atinge mais de $20 \%$ da população e encontra-se em crescimento na 
maioria dos países. É responsável por 7,1 milhões de mortes, correspondendo a 13\% do total (SESA, 2008).

O perfil alimentar predominantemente industrializado, com excesso de sal, de gorduras animais e o aumento da obesidade vêm aumentando as taxas de hipertensão arterial, bem como o sedentarismo, tabagismo, estresse e alcoolismo (SESA, 2008).

\subsubsection{Prevalência da hipertensão em Curitiba}

Os dados apresentados são resultado de um inquérito que identificou os indivíduos que informaram ter medido a pressão arterial nos últimos dois anos. Este inquérito identificou que entre os 2.337 entrevistados, 1.649 pessoas passaram por tomada de medida pressórica nos dois anos que antecederam o inquérito e 25,7\% foram informados de que eram hipertensos. Dados do Inquérito Nacional colocam a cidade de Curitiba como a 8aㅗ maior taxa de hipertensão entre as capitais brasileiras (SESA, 2008).

\subsection{Macadamia e a indústria}

Ao natural, a noz de macadâmia é excelente na preparação de pratos doces e salgados, acompanhamento de carnes, na fabricação de sorvetes, na indústria de chocolate, entre outros. É utilizada, em menor escala, na indústria de cosméticos devido ao seu óleo ser rico nos ácidos graxos insaturados: linoléico e linolênico, o que faz com que seja lubrificante para a pele e mantenha seu o teor hídrico. O óleo também pode ser comestível (BEUCHAT; WORTHINGTON, 2007).

\section{MATERIAL E MÉTODOS}

\subsection{Material}

A amostra de macadâmia (Macadamia integrifolia Maiden \& Betch) foi adquirida sem sal ou torrefação, embalada à vácuo, no comércio varejista de Curitiba, em agosto de 2010.

\subsection{Método}

\subsubsection{Análises químicas e nutricionais}

Para a realização das análises da amostra de macadâmia foram realizadas as 
análises a seguir:

- Determinação da umidade (IAL, 2008)

- Determinação do nitrogênio total - método de Micro Kjeldahl (AOAC, 1970), utilizando fator de conversão 5,30 (FREITAS; NAVES, 2010)

- Determinação de lipídios (IAL, 2008)

- Determinação de polissacarídeos (AOAC, 1970)

- Determinação de minerais (IAL, 2008)

- Determinação de carboidratos por dferença, obtendo fração NiFext.

\section{RESULTADOS E DISCUSSÃO}

TABELA 1: RESULTADOS OBTIDOS NA COMPOSIÇÃO CENTESIMAL E NUTRICIONAL DA (Macadamia integrifolia Maiden \& Betch)

\begin{tabular}{cccc}
\hline Determinação & g em 100g & g em 15g (1 colher de sopa) & VD\% \\
\hline Umidade & $7,33( \pm 0,54)$ & 1,10 & - \\
Proteína & $9,32( \pm 0,37)$ & 1,40 & 1,85 \\
Lipídeos & $70,39( \pm 0,53)$ & 10,56 & 13,57 \\
Carboidratos & 7,31 & 1,09 & 0,44 \\
Fibras & $4,54( \pm 0,29)$ & 0,68 & 2,43 \\
Minerais & $1,10( \pm 0,13)$ & 0,16 & - \\
Kcal & 700,08 & 105,01 & 5,25 \\
\hline
\end{tabular}

Fonte: Os autores, 2010

Dentre as determinações para a composição centesimal, determinação de umidade é uma das medidas mais importantes e utilizadas na análise de alimentos. Sua quantidade implica diretamente na conservação do alimento, estando relacionada com sua estabilidade, qualidade e composição, e pode afetar diretamente a estocagem, a embalagem e o processamento do alimento.

De acordo com a literatura, o teor de umidade para a macadâmia (Macadamia integrifolia Maiden \& Betch) varia na faixa de 1,2 a 5,5\% (SILVA, 2003), e 2,10\% (FREITAS; NAVES, 2010) não estando condizente com o nosso resultado que foi de $7,33 \%$ de umidade. Esta comparação pode ser melhor visualizada na Figura 1. 


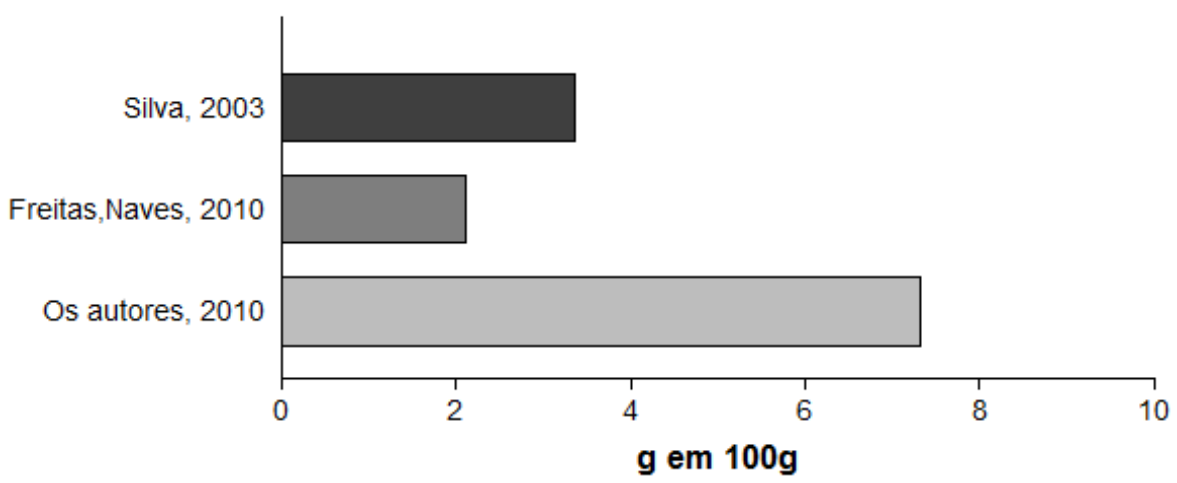

FIGURA 1: COMPARAÇÃO ENTRE VALORES DE UMIDADE DA NOZ MACADAMIA

Mas segundo Cecchi (2003), a exatidão e a precisão do método tornam-se complicadas devido a algumas dificuldades encontradas como a separação incompleta da água do alimento, decomposição do alimento com formação de água além da original ou perda das substâncias voláteis do alimento que serão computadas como peso em água.

O valor de umidade de uma amostra está diretamente relacionado à possibilidade de contaminação dessa por microorganismos. Se, por exemplo, o valor de umidade de um alimento estiver fora dos padrões recomendados, podem ocorrer diversos tipos de contaminação, como a contaminação fúngica, que é um aspecto muito importante na análise de alimentos, especialmente os grãos. Esse problema se torna fundamental quando é observada a produção de metabólitos tóxicos - micotoxinas - por alguns fungos como, por exemplo, os dos gêneros Aspergillus, Penicillium e Fusarium.

Segundo a European Mycotoxin Awareness Network, as aflatoxinas são de extrema importância tendo em vista o seu potencial carcinogênico, sendo a aflatoxina B1 um dos agentes hepato-carcinogênicos mais potentes conhecidos. Além disso, a mistura das aflatoxinas B1, B2, G1 e G2 são classificadas como carcinogênicas para humanos (IARC, 2002). No Brasil, o nível máximo permitido pela legislação é de $20 \mu \mathrm{g} / \mathrm{Kg}$ para a soma das aflatoxinas $\mathrm{B} 1+\mathrm{G} 1+\mathrm{B} 2+\mathrm{G} 2$ (ANVISA, 2002).

$\mathrm{Na}$ amostra utilizada, conforme análises provenientes do produtor (ANEXO 1), não foram encontrados valores relevantes de aflatoxinas.

Além dos valores de umidade, a determinação do conteúdo de proteínas em uma amostra é extremamente necessária, visto essas são macronutrientes e, por isso, sua quantidade e qualidade em um alimento podem determinar se esse pode constituir-se em uma boa fonte energética.

O método utilizado nessa pesquisa determina o nitrogênio total da amostra, sendo necessária a conversão do valor para o teor de proteínas. Para nozes e sementes 
comestíveis, o fator comumente utilizado é 5,30 (FREITAS; NAVES, 2010).

Durante os testes, o teor de proteínas determinado foi 9,32\%, relativamente próximo aos valores encontrados na literatura, 8,40 (FREITAS; NAVES, 2010), 7,2 1 10,2 (SILVA, 2003). Isso pode ser observado da figura 2.

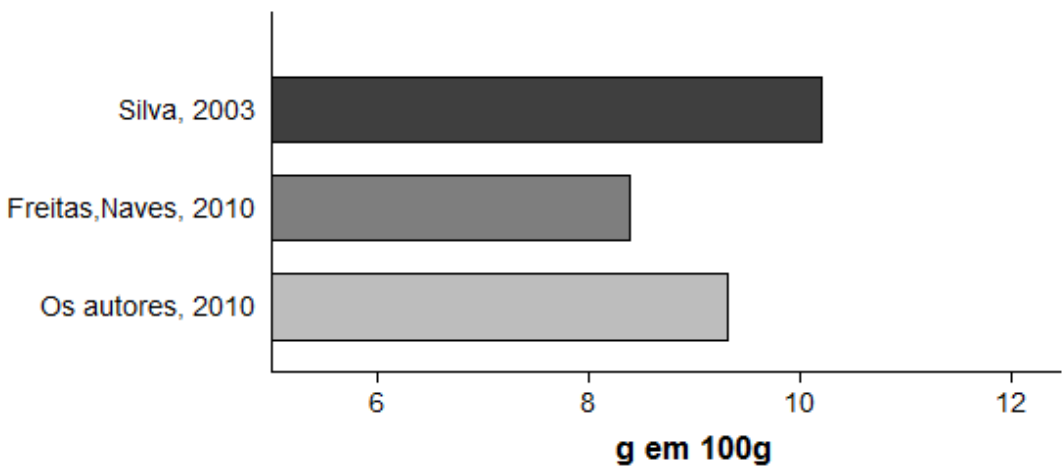

FIGURA 2: COMPARAÇÃO ENTRE VALORES DE PROTEÍNA

A avaliação quantitativa de lipídeos presentes na amostra é de vital importância, posto que as características específicas desses óleos refletem em diversas aplicações farmacológicas, seja na indústria de cosméticos como na área alimentícia.

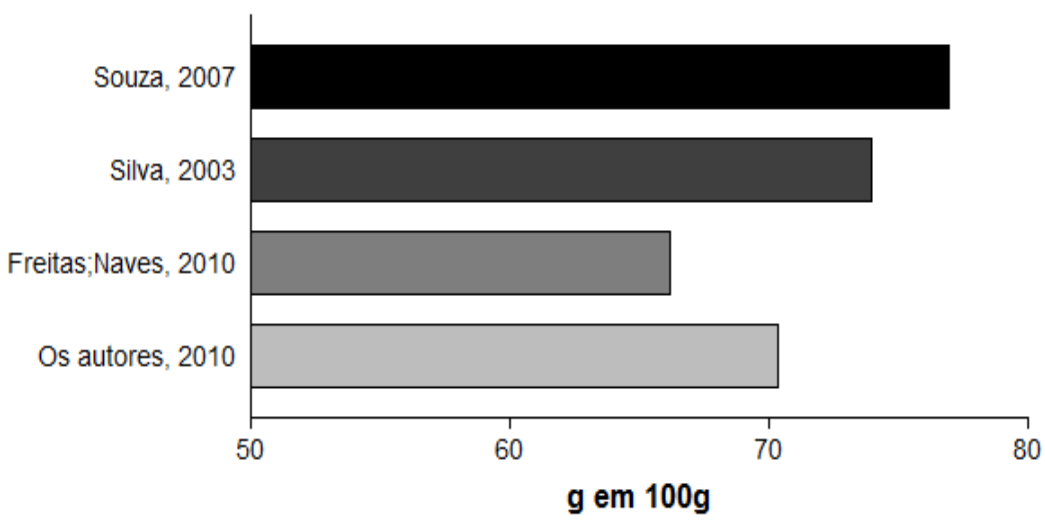

FIGURA 3:COMPARAÇÃO ENTRE VALORES DE LIPÍDEOS

Para realizar essa avaliação optou-se pelo método de extração com éter. A média dos resultados obtidos (70.39\%), indicados na Figura 3, apresentou pequena variação com a literatura: 74,19 a 79,77\% segundo Souza (2007), 70,00 a 78\% segundo Silva (2003) e 66,16\% (FREITAS, NAVES, 2010), sendo que essa divergência encontra-se no esperado devido às variações que a noz sofre provocadas por clima, sazonalidade, umidade e outros 
fatores ambientes originais do local de cultivo e que interferem diretamente no desenvolvimento da noz.

Esses resultados atestam a riqueza da noz da macadâmia em óleos, cujo alto valor agregado deve-se às propriedades físico-químicas e nutricionais apresentadas.

Em relação a composição centesimal, a determinação de carboidratos foi realizada por diferença. $O$ valor encontrado no experimento foi de $7,31 \%$ o que não condiz com os valores da literatura, como o de Freitas, Naves (2010), que foi 22,18\%, os de Silva (2003), de 8,4 à 16,7\%, e o de Philuppi (2003), 13,70\%. Isso se deve à procedência da noz, que causou alteração nos valores da composição centesimal.

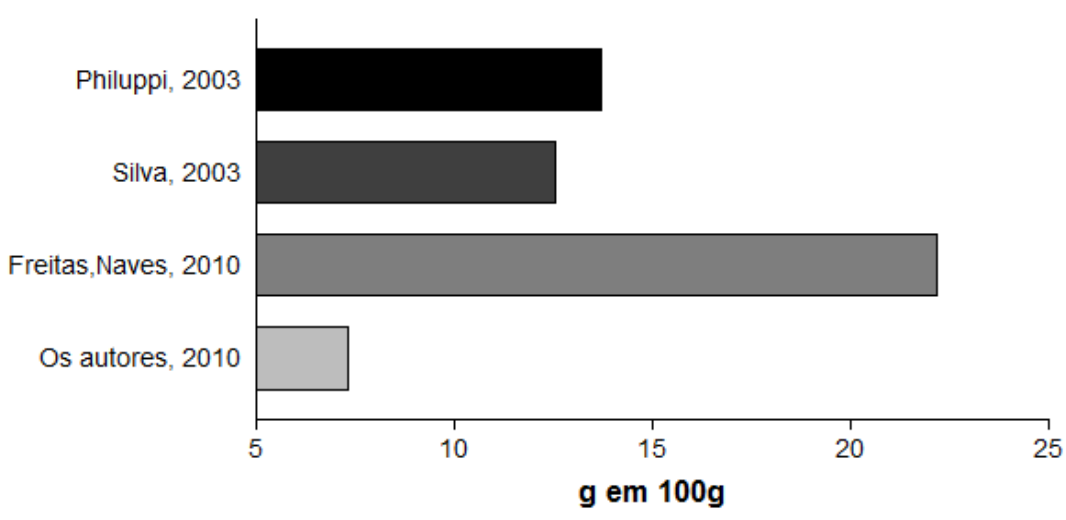

FIGURA 4: COMPARAÇÃO ENTRE TEORES DE CARBOIDRATOS

Assim como nos carboidratos, o valor de fibra bruta na amostra, determinada por meio de digestão ácida $\left(\mathrm{H}_{2} \mathrm{SO}_{4}-1,25 \%\right)$ e básica $(\mathrm{NaOH}-1,25 \%)$ resultando em um resíduo orgânico (fibras), 4,54\%, destoou dos valores encontrados na literatura, 8,00\% (GARBELINI, 2009) e 5,31\% (PHILUPPI, 2003).

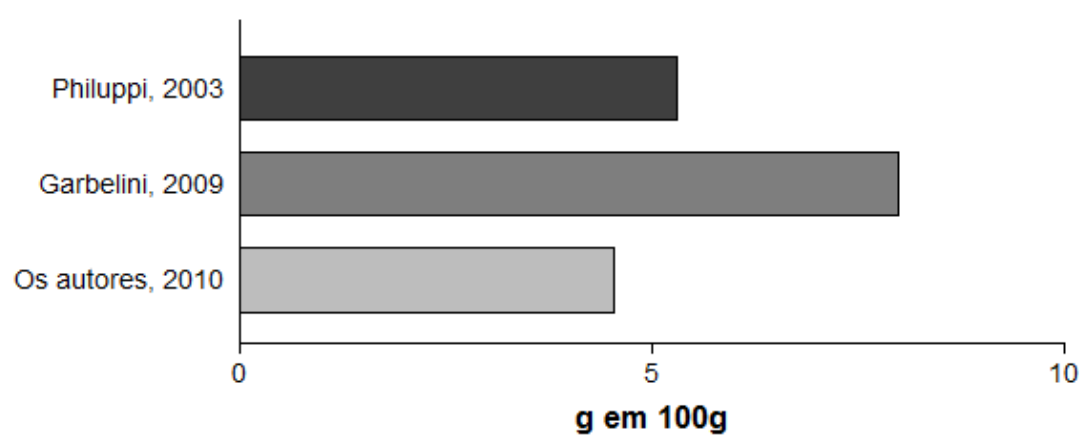

FIGURA 5: COMPARAÇÃO ENTRE VALORES DE FIBRA BRUTA. 
De acordo com Cecchi (2003), o conteúdo de cinzas totais para determinação de minerais para nozes, como é classificada a macadâmia varia entre $1,7 \%$ a 3,6\%, estando diferente do valor obtido da nossa amostra que foi de 1,1\%. As justificativas para as diferenças na composição podem apontar como fatores: a natureza do alimento, o local de crescimento, a variedade, os tratos culturais, a época de crescimento e a natureza do método de determinação utilizado. Porém, o valor citado por Freitas, Naves (2010): 1,16\%, se aproxima muito mais do valor obtido em nossos experimentos.

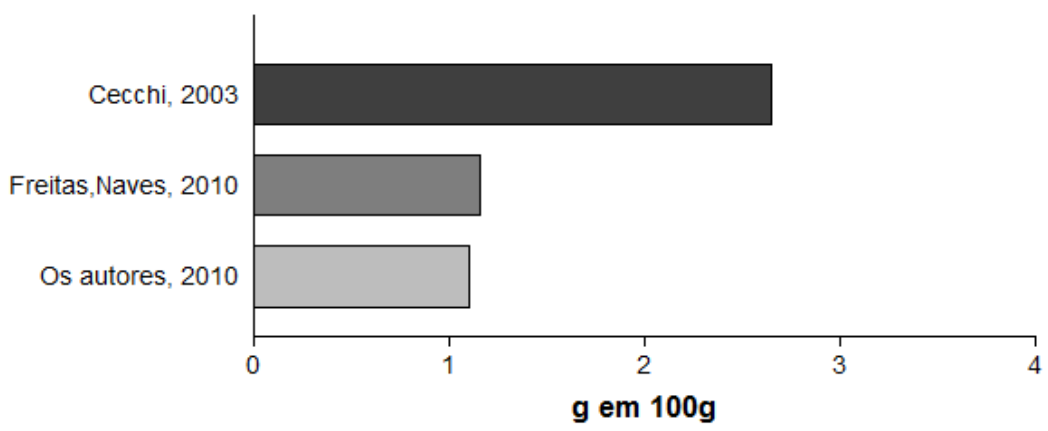

FIGURA 6:

\section{CONCLUSÃO}

As análises realizadas apresentaram resultados muito semelhantes aos dados obtidos na literatura, apenas com variações consideradas aceitáveis. Dessa forma, é possível aferir que, no caso do perfil lipídico das macadâmias (Macadamia integrifolia Maiden \& Betch), questão mais importante relacionada à terapêutica da noz e o seu uso como alimento funcional, a amostra utilizada deve apresentar relação semelhante de ácidos graxos $\omega-6: \omega-3$, tendo, portanto, funcionalidade na redução dos riscos de patologias cardiovasculares.

\section{REFERÊNCIAS}

ANVISA - AGÊNCIA NACIONAL DE VIGILÂNCIA SANITÁRIA. Resolução № 3480. Disponível em: <http://portal.saude.gov.br/portal/arquivos/pdf/portaria79.pdf>. Acesso em 15/10/2010. AOAC - ASSOCIATION OF OFFICIAL AGRICULTURAL CHEMISTS. Official methods os analysis. 12 Ed. Washington D.C.: Association of Official Agricultural Chemists, 1970. 
BEUCHAT, L.R.; WORTHINGTON, R.E. Technical note: Fatty acid composition of tree nut oils. International Journal of Food Science \& Technology, vol.13, n.4, p 355-358, 2007. Disponível em: <http://onlinelibrary.wiley.com/doi/10.1111/j.1365-2621.1978.tb00813.x/ abstract>. Acesso em: 27/08/2010.

BOSCO, S.M.D.Efeito do ácido graxo poliinsaturado ômega-3 em pacientes obesos mórbidos e com síndrome metabólica. 83 f. Tese (Doutorado em Medicina e Ciências da Saúde) - Pontifícia Universidade Católica do Rio Grande do Sul. Porto Alegra, 2009. Disponível em: <http://tede.pucrs.br/tde_busca/arquivo. php?codArquivo=2412>. Acesso em: 30/08/2010.

CECCHI, H.M. Fundamentos teóricos e práticos em análise de alimentos. Campinas: Editora da Unicamp, 2003. Disponível em: http://issuu.com/editorauni camp/docs/571?mode=a_p. Acesso em: 01/11/2010.

DIERBERGER, J.E.; NETTO, L.M. Noz macadamia: uma nova opção para a fruticultura brasileira. São Paulo: Livraria Nobel, 1985. Disponível em:<http://books.google.com.br/ books? $i d=b d P s t 4 c f t Q Q C \& p g=P A 29 \& d q=c o m p o s i \% C 3 \% A 7 \% C 3 \% A 30+d o s+$ carboidratos+ na + macad\%C3\%A2mia\&lr=\&source $=g b s \_t o c \_r \& c a d=4 \# v=0 n e p a g e \& q \& f=f a l s e>. \quad$ Acesso em: 26/08/2010.

EMAN - EUROPEAN MYCOTOXINS AWARENESS NETWORK: The Aflatoxins. Disponível em: http://services.leatherheadfood.com/mycotoxins/item.asp? sectionid=1\&mytype=basic \&number=2\&fsid=6. Acesso em: 15/10/2010.

FREITAS, J.B.; NAVES, M.M.V. Composição química de nozes e sementes comestíveis e sua relação com a nutrição e saúde. Revista de Nutrição, vol. 23, n. 2, p. 269-279, 2010. Disponível em: <http://www.scielo.br/scielo.php?script=sci_arttext\&pid=S1415-527320100 00200010\&lng=pt\&nrm=iso\&tlng=pt/>. Acesso em: 16/08/2010.

GARBELINI, R.C.B.S. Reguladores vegetais na emergência e no desenvolvimento de plantas de macadâmia (Macadamia integrifolia Maiden \& Betche). 94 f. Tese (Doutorado em Ciências Biológicas - Botânica) - Instituto de Biociências, Universidade Estadual Paulista "Julio de Mesquita Filho", Botucatu, 2009. Disponível em: 


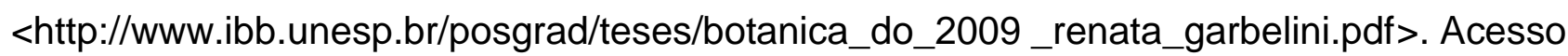
em: 02/11/2010.

GOGOS, C.A.; SKOUTELIS, A.; KALFARENTZOS, F. The effects of lipids and the imunne response of patients with cancer. Jornal of Nutrition Health and Aging., vol.4, n.3., p 1725, 2000. Disponível em: <http://www.ncbi.nlm.nih.gov/pubmed/10936906>. Acesso em: $24 / 08 / 2010$.

IAL - INSTITUTO ADOLFO LUTZ Normas Analíticas do Instituto Adolfo Lutz, 2008. Disponível em: <http://www.ial.sp.gov.br/index.php?option=com_remository\&ltemid=7\& func=select\&orderby=1\&ltemid=7>. Acesso em: 30/08/2010.

IARC - International Agency of Research on Cancer - Monographs on the evaluation of carcinogenic risks to humans. Lyon: OMS, v. 82, 2002. Disponível em: <http://monogra phs.iarc.fr/ENG/Monographs/vol82/mono82.pdf)>. Acesso em: 16/10/2010.

LIMA, F et al. Ácidos graxos e doenças cardiovasculares: uma revisão. Revista de nutrição, Campinas, vol. 13, n 2, p. 73-80, 2000. Disponível em: <http://www.scielo.br/ scielo.php?pid=S141552732000000200001\&script=sci_arttext>. Acesso em: 24/08/2010

PAULINO, F. D. G.; MARCHINI, L. C. Insetos associados às panículas de macadâmia (Macadamia integrifólia Maiden \& Betche). Scientia Agricola, vol.55, n.3, 1998. Disponível em: $<$ http://www.scielo.br/scielo.php?scrip =sci_arttext\&pid=S0103-90161998000300023>. Acesso em 30/08/2010.

PHILUPPI, S.T. Nutrição e Técnica Dietética. 1. ed. Barueri: Editora Manole, 2003.

QUEEN NUT MACADAMIA. Disponível em: <http://www.queennutmacadamia.com. $\mathrm{br} /$ ?conteudo=tratos_culturais $>$. Acesso em: 23/08/2010.

SANTOS, L.E.S.; BORTO-LOZO, E.A.F.Q. Ingestão de ômega-3: considerações sobre potencias benefícios no metabolismo lipídico. Publicatio UEPG - Ciências Exatas e da Terra, Agrárias e Engenharias, vol. 14, n. 2, p. 161-170, 2008. Disponível em: 
<http://www.revistas2.uepg.br/index.php/exatas/article/viewFile /782/688> . Acesso em: 25/08/2010.

SACRAMENTO, C.K. et al. Capacidade combinatória para frutificação em cultivares de nogueira macadâmia. Pesquisa agropecuária brasileira, vol.34, n.11, p 2045-2049, 1999. Disponível em: <http://www.scielo.br/scielo.php?pid=S0100-204X1999001100010\&script= sci_abstract\&tlng=pt>. Acesso em 02/09/2010.

SESA - SECRETARIA DE ESTADO DA SAÚDE DO PARANÁ - SUPERINTENDÊNCIA DE VIGILÂNCIA E SAÚDE. Doenças e agravos não transmissíveis no estado do Paraná. Paraná, 2008. Disponível em:<http://www.saude.pr.gov.br/arquivos/File/vig_epide miolog/DVDNT/DANT S/PARTE3.pdfv>. Acesso em: 01/09/2010.

SILVA, M.G. Macadâmia nacional: tocoferóis e caracterização físico-química. Dissertação (Mestrado em Ciência dos Alimentos) - Faculdade de Engenharia de Alimentos, Unicamp, Campinas, 2003. Disponível em: <http://www.fea.unicamp.br /alimentarium/ver_documento.php?did=427>. Acesso em 01/11/2010.

SOUZA, D.F.S. et al. Estabilidade oxidativa dos óleos de macadâmia e pistache. Boletim do Centro de Pesquisa de Processamento de Alimentos, Curitiba, v. 25, n. 1, p. 141156, 2007. Disponível em:<http://ojs.c3sl.ufpr.br/ojs2/index.php/a imentos/article/view/8402 /5851>. Acesso em 02/11/2010. 
ANEXO 1

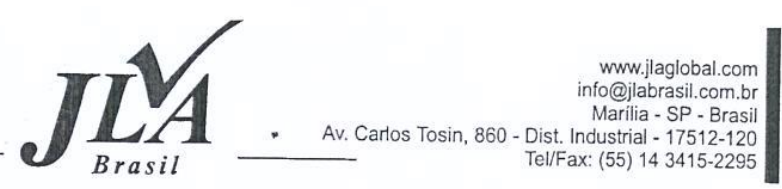

RELATORIO de RESULTADOS

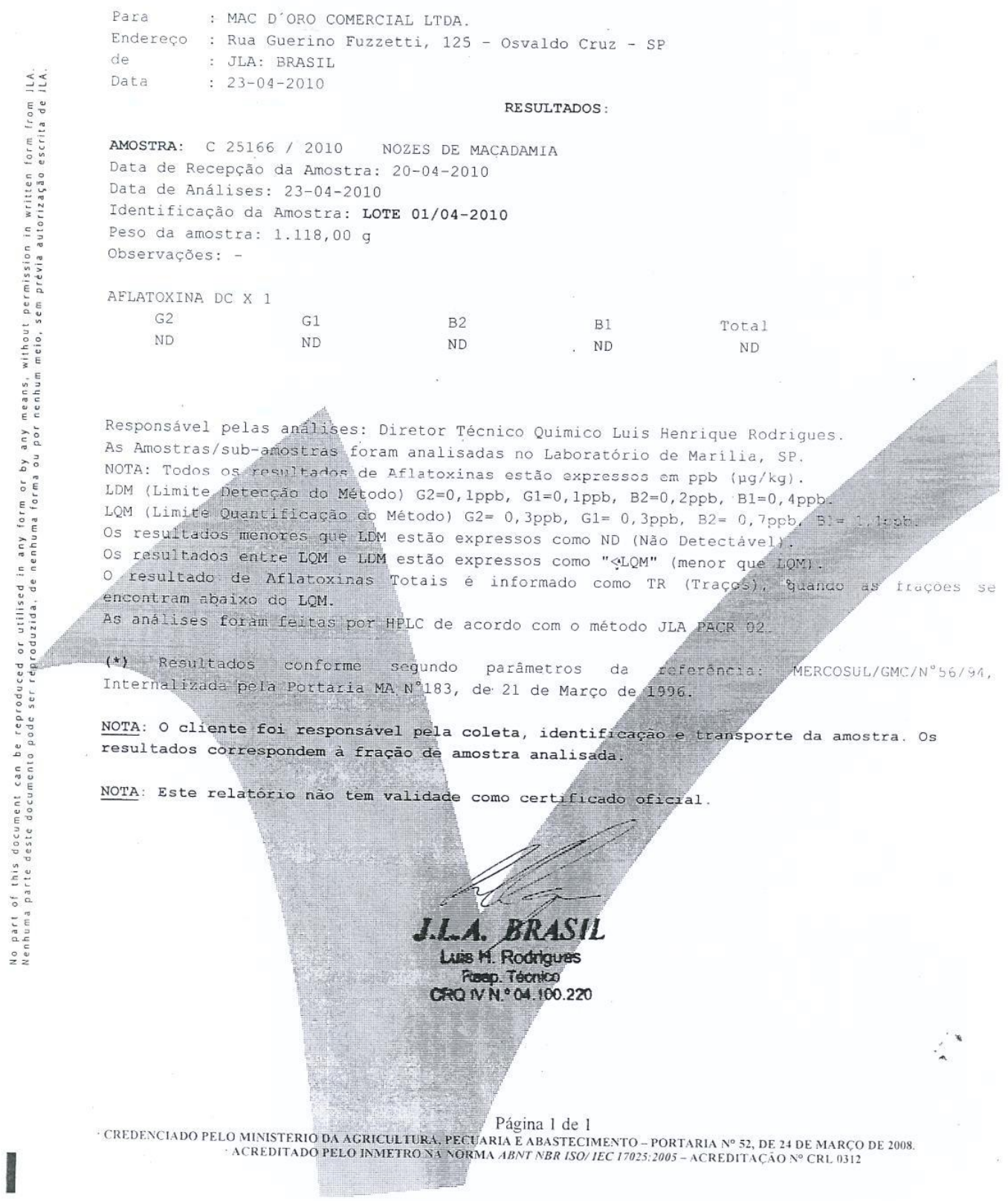

\section{ANEXO 2}




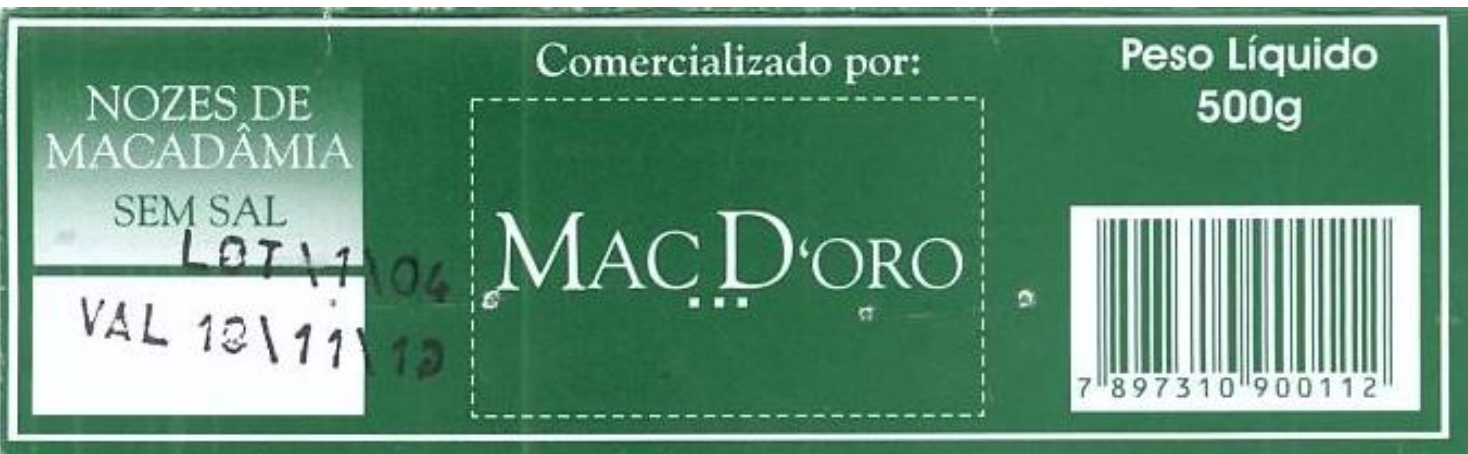

PROCESSADO E EMBALADO POR:

MAC D ORO COMERCIAL LTDA.

CNPJ: $00.414 .602 / 0001.95$

L.E: 494.022 .043 .115

www.macdoro.com.br

Fone(18) $3528-3536$

Contém:

Macadâmia

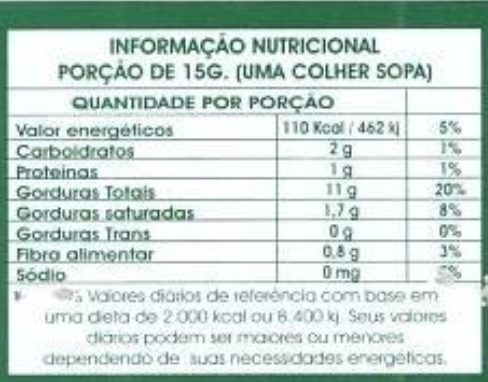

PRODUTO NATURAL

SEM CONSERVANTES

SEM COLESTEROL RICO EM FIBRAS

EMBALAGEM À VACUO

"Recomenda-se consumir em 7 dias após abrir a embalagem 